\title{
Défice de Vitamina D e Demência - Qual a Associação?
}

Maria Margarida Baldaque de Sousa Soares da Silva Oliveira, Maria Inês Monteiro da Silva Dinis Capitão, Joana Maria Pedrosa Coelho, Fernando José Lima Fernandes

\section{Resumo}

Introdução: A vitamina D é considerada uma hormona esteroide major que, para além do seu reconhecido papel no metabolismo ósseo, poderá estar envolvida no funcionamento cerebral e na neuroprotecção. Estudos recentes parecem indicar que a deficiência da vitamina D é mais prevalente em indivíduos com demência e que pode constituir um fator de risco potencial para o seu desenvolvimento. 0 objetivo deste trabalho foi rever a evidência científica mais atual sobre a associação entre o défice da vitamina $\mathrm{D}$ e a demência.

Metodologia: Pesquisa bibliográfica de metanálises (MA), revisões sistemáticas (RS), normas de orientação clínica e estudos observacionais, em sites de medicina baseada na evidência, publicados nos últimos 10 anos, utilizando os termos MeSH: "vitamin D" e "dementia". Foram selecionados os artigos nas línguas portuguesa, espanhola e inglesa. Para atribuição do nível de evidência (NE) foi utilizada a escala Strenght of Recommendation Taxonomy (SOR) da American Academy of Family Physicians.

Resultados: Obtiveram-se 370 artigos. Treze satisfizeram os critérios de elegibilidade: 5 revisões sistemáticas (RS), 4 delas com metanálise, 3 estudos de coorte prospetivo e 3 estudos transversais. Na maioria das publicações incluídas verificou-se que a deficiência de vitamina D é mais prevalente em indivíduos com demência e que está associada ao risco de desenvolvimento da doença.

Conclusões: A evidência disponível mostra uma associação direta entre o défice de vitamina $D$ e a demência (SOR B). Parece haver associação positiva entre o défice de vitamina $\mathrm{D}$ e o risco de desenvolvimento de demência (SOR B).

\begin{abstract}
Introduction: Vitamin D is considered a major steroid hormone, that in addition to its well-established role on the bone metabolism may be also involved in the brain functioning and neuro-protection. Recent studies suggest that vitamin D insufficiency is more prevalent in people with dementia and that may be a risk factor to its development. The goal of this study is to review the recent scientific evidence associating vitamin $D$ deficiency and dementia.
\end{abstract}

Methods: Literature search of meta-analysis, systematic reviews, guidelines and observational studies, in evidence based medicine sites, published in the last 10 years, using the MeSH terms: "vitamin D", "dementia" and "Alzheimer disease". We selected articles written in portuguese, spanish and english. To attribute the level of evidence we used the Strength of Recommendation Taxonomy (SOR) scale from the American Academy of Family Physicians.

Results: We collected 370 articles. 13 filled the criteria for eligibility: 5 systematic reviews - 4 of them with meta-analysis, 3 prospective cohort studies and 3 transversal studies. In the majority of the included publications it was concluded that the deficiency in vitamin $D$ is more prevalent in individuals with dementia and that this deficiency is associated with the risk of development of the disease.

Conclusion: The available evidence shows a direct association between the deficit of vitamin $D$ and dementia (SOR B). There seems to be a positive association between the deficiency of vitamin $D$ and the risk of developing dementia (SOR B). 
Será importante a realização de ensaios clínicos prospetivos, bem desenhados, randomizados e controlados que permitam avaliar a existência de causalidade, bem como a eficácia da suplementação de vitamina D em populações com hipovitaminose D.

Palavras-chave: vitamina D, demência, doença de Alzheimer
It will be important to perform prospective clinical trials - well designed, controlled and randomized - that would allow to determine causality, as well as the efficacy of supplementing with vitamin D populations with hypovitaminosis D.

MeSH terms: vitamin D, dementia, Alzheimer disease

\section{Introdução}

Segundo dados de 2010, existem cerca de 24.3 milhões de pessoas com demência em todo o mundo e o número irá duplicar a cada 20 anos, pelo que é importante identificar fatores de risco modificáveis para o desenvolvimento de demência, incluindo fatores relacionados com o estilo de vida e dieta. ${ }^{1,2}$

A vitamina D é considerada uma hormona esteróide major que, para além do seu reconhecido papel no metabolismo ósseo, poderá estar envolvida no funcionamento cerebral e na neuroproteção. ${ }^{3}$ As numerosas funções da vitamina $D$ no sistema nervoso incluem a regulação da produção do fator neurotrófico, a libertação de neurotransmissores, a homeostasia do cálcio, os mecanismos do stress oxidativo, a modulação do sistema imune e do processo inflamatório e a estimulação da fagocitose do péptido beta-amilóide. - $^{-9}$ Sabe-se que estes processos estão alterados durante o enveIhecimento e na demência, nomeadamente na Doença de Alzheimer (DA). ${ }^{10}$

O estado nutricional de vitamina $D$ é refletido pelas concentrações séricas 25 hidroxi-vitamina D $(25(\mathrm{OH})$ D). De acordo com a Endocrine Society, o défice de vitamina $\mathrm{D}$ é definido como níveis de vitamina $\mathrm{D}$ abaixo de $20 \mathrm{ng} / \mathrm{ml}$ (50 nmol/L) e insuficiência de vitamina D entre $21-29 \mathrm{ng} / \mathrm{ml}(52.5-72.5 \mathrm{nmol} / \mathrm{L}) .{ }^{11}$ A carência de vitamina D é prevalente em todo o mundo, pensando-se que atinja cerca de um milhão de indivíduos. Trata-se de um problema transversal a todas as idades nos países ocidentais, mas também, na Asia e Médio Oriente e que tem vindo a aumentar. Estima-se que nos Estados Unidos da América, 34\% da população adulta entre os 18 e os 65 anos apresente défice de vitamina D. ${ }^{12}$ Em Portugal, não existem estudo epidemiológicos acerca da prevalência de valores inadequados de vitamina D. Todavia, vários estudos europeus mostram proporções importantes desta carência, sendo expectável que a realidade portuguesa seja semelhante. ${ }^{13-15}$
Níveis baixos de vitamina $\mathrm{D}$ parecem constituir um fator de risco potencial para o desenvolvimento de DA e outras demências, tornando a vitamina $D$ uma potencial ferramenta terapêutica e preventiva. ${ }^{10,16.17}$ Deste modo, este estudo teve como objetivo rever a evidência científica mais atual sobre a associação entre o défice da vitamina D e a demência.

\section{Métodos}

Foi realizada uma pesquisa bibliográfica em setembro de 2016, utilizando os termos MeSH: “vitamin D" e "dementia”. Foi efetuada a pesquisa de metanálises (MA), revisões sistemáticas (RS), normas de orientação clínica e estudos originais, nas fontes de dados National Guideline Clearinghouse, Guidelines Finder, TRIP Database, Cochrane Library, DARE (Database of Abstracts of Reviews of Effectiveness), Evidence Based Medicine Online e Pubmed. A pesquisa foi limitada aos artigos publicados entre janeiro de 2006 e setembro de 2016. Para garantir a atualidade da revisão, foram procurados artigos originais publicados nos últimos dois anos na Pubmed, utilizando a opção "Clinical Queries". Foram selecionados os artigos nas línguas portuguesa, espanhola e inglesa, que incluíam indivíduos adultos, acima dos 18 anos e sem limite superior de idade cujos niveis de vitamina $D$ foram determinados. 0 outcome avaliado foi a associação entre o défice de vitamina $D$ e demência.

Foram excluídos artigos repetidos ou que não cumpriam os critérios de inclusão no que se refere à população e outcome, nomeadamente os que se referiam só a défice/declínio cognitivo.

Para atribuição do nível de evidência (NE) foi utilizada a escala Strenght of Recommendation Taxonomy (SOR) da American Academy of Family Physicians. 


\section{Resultados}

A pesquisa por termos MESH resultou num total de 370 artigos. Dezassete foram excluídos por serem repetidos, 316 foram excluídos por se encontrarem fora do objetivo do trabalho, pela língua de redação, tipo de artigo ou não ser possível a sua obtenção após contacto com os autores. Trinta e sete foram selecionados para leitura integral, dos quais 11 foram incluídos e agrupados de acordo com o tipo de artigo: 5 revisões sistemáticas (RS), 4 delas com metanálise (MA), 3 estudos de coorte prospetivo, 3 estudos transversais. (Figura1)
Em muitos dos artigos selecionados era avaliada, no mesmo artigo, a associação entre os níveis de vitamina $\mathrm{D}$ e a função cognitiva, défice cognitivo ligeiro (DLC) e demência. Os autores desta revisão, tendo por base o seu objetivo, apresentam apenas os resultados que associam os níveis de vitamina $D$ e demência.

Figura 1. Fluxograma da seleção dos estudos.

\section{Total de artigos obtidos:} 370 artigos

353 artigos após exclusão dos repetidos

35 artigos sujeito a leitura integral

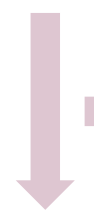

11 artigos incluídos

\section{8 artigos excluídos:}

- 307 fora do âmbito desta revisão

- 2 pela língua de redação (um italiano e um alemão)

- 5 pelo tipo de artigo (comentário, carta ao editor, revisão clássica, opinião de peritos)

- 4 não foi possível obter o artigo integral (mesmo após contacto com o autor por email)

24 artigos completos excluídos:

- 14 artigos fora do âmbito do trabalho

- 10 artigos já incluídos em artigos selecionados 
Quadro 1. Revisões sistemáticas analisadas.

\section{Revisões sistemáticas e metanálises:}

\begin{tabular}{|c|c|c|c|c|c|}
\hline $\begin{array}{l}\text { Tipo de } \\
\text { estudo }\end{array}$ & Referências & Objetivo do estudo & População/Intervenção & Conclusões & NE \\
\hline 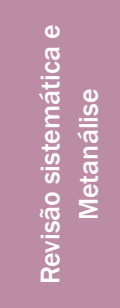 & $\begin{array}{l}\text { Annweiler } \\
\text { et al } \\
2013\end{array}$ & $\begin{array}{l}\text { Rever a evidência } \\
\text { que associa } \\
\text { concentrações } \\
\text { séricas baixas de } \\
\text { vit. D e DA }\end{array}$ & $\begin{array}{l}\text { - } 10 \text { estudos: } 9 \text { casos-controlo (dos } \\
\text { quais } 7 \text { foram sujeitos a MA) e } 1 \\
\text { coorte } \\
\text { - } n=440 \text { (casos); } n=786 \text { (controlos) } \\
\text { MA: } n=357 \text { (casos) e } n=648 \\
\text { (controlos) } \\
\text { - Maioria do sexo feminino, idades: } \\
66-81 \text { anos }\end{array}$ & $\begin{array}{l}\text { Os casos de DA } \\
\text { apresentaram } \\
\text { concentrações séricas } \\
\text { de vit. D inferiores aos } \\
\text { controlos }\end{array}$ & 2 \\
\hline 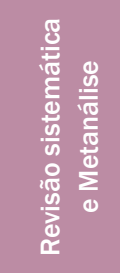 & $\begin{array}{l}\text { Cao et al } \\
2015\end{array}$ & $\begin{array}{l}\text { Avaliar a } \\
\text { associação entre a } \\
\text { dieta mediterrânica } \\
\text { e alguns } \\
\text { componentes da } \\
\text { dieta e o risco de } \\
\text { demência }\end{array}$ & $\begin{array}{l}\text { - } 3 \text { estudos prospectivos } \\
\text { - Follow-up } 5.6 \text { a } 21 \text { anos }\end{array}$ & $\begin{array}{l}\text { Baixos níveis de vit. } \\
\text { D foram associados } \\
\text { a declínio cognitivo e } \\
\text { demência }\end{array}$ & 2 \\
\hline 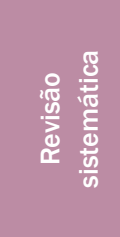 & $\begin{array}{l}\text { van der } \\
\text { Schaft et al } \\
2013\end{array}$ & $\begin{array}{l}\text { Avaliar a associação } \\
\text { entre os níveis } \\
\text { séricos de vit. D e a } \\
\text { cognição }\end{array}$ & $\begin{array}{l}\text { - } 22 \text { estudos transversais ( } n=93921 \text {; } \\
20-97 \text { Ano) } \\
\text { - } 6 \text { estudos prospectivos ( } n=10896 \text {; } \\
\geq 65 \text { Anos; follow-up } 4 \text { a } 7 \text { Anos) } \\
\text { - Níveis de Vit. D (doseamento, ingestão } \\
\text { na dieta ou suplementação) }\end{array}$ & $\begin{array}{l}\text { A hipovitaminose D está } \\
\text { associada a um pior } \\
\text { resultado em um ou mais } \\
\text { testes de função cognitiva } \\
\text { ou com uma maior } \\
\text { frequência de demência }\end{array}$ & 2 \\
\hline 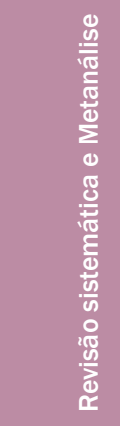 & $\begin{array}{l}\text { Lopes da } \\
\text { Silva et al } \\
2014\end{array}$ & $\begin{array}{l}\text { Comparar os níveis } \\
\text { plasmáticos de } \\
\text { micronutrientes e } \\
\text { ácidos gordos em } \\
\text { pacientes com DA e } \\
\text { em controlos idosos } \\
\text { sem alterações } \\
\text { cognitivas e que } \\
\text { não difiram na } \\
\text { ingestão diária de } \\
\text { proteínas e energia }\end{array}$ & $\begin{array}{l}\text { - } 5 \text { RS com MA } \\
\text { - } \mathrm{n}=394 \text { (casos) e } \mathrm{n}=471 \text { (controlos) }\end{array}$ & $\begin{array}{l}3 \text { estudos não mostraram } \\
\text { diferença estatística nos } \\
\text { níveis de vit. D nos doentes } \\
\text { com DA face aos controlos; } \\
2 \text { estudos mostraram } \\
\text { níveis mais baixos de vit. D } \\
\text { nos doentes com DA face } \\
\text { aos controlos }\end{array}$ & 2 \\
\hline 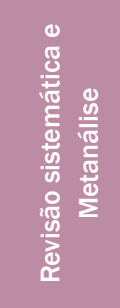 & $\begin{array}{l}\text { Shen and Ji } \\
2015\end{array}$ & $\begin{array}{l}\text { Estimar a } \\
\text { associação entre } \\
\text { a deficiência de } \\
\text { vit. D e risco de } \\
\text { desenvolver DA e } \\
\text { demência }\end{array}$ & $\begin{array}{l}\text { - } 2 \text { estudos prospetivos e } 3 \text { estudos } \\
\text { transversais } \\
\text { - } n=25157\end{array}$ & $\begin{array}{l}\text { Indivíduos com deficiência } \\
\text { de vit. } D(\leq 50 \mathrm{nmol} / \mathrm{L}) \\
\text { apresentaram um } \\
\text { risco maior para o } \\
\text { desenvolvimento da DA e } \\
\text { demência em comparação } \\
\text { com aqueles com níveis > } \\
50 \mathrm{nmol} / \mathrm{L}\end{array}$ & 2 \\
\hline
\end{tabular}

Três das $\mathrm{RS}^{18-20}$, as duas primeiras com MA, concluíram que níveis séricos baixos de vitamina D estão associados a DA ou demência. Lopes da Silva et al (2014) ${ }^{21}$ realizaram uma RS com MA, que incluiu outras 5 RS com MA em que foi avaliada a associação entre os níveis de vitamina D e a DA. Destas, 3 não mostraram diferença estatisticamente significativa nos níveis de vitamina D nos doentes com DA face aos controlos e duas mostraram níveis de vitamina $D$ mais baixos nos doentes com DA face aos controlos.

Shen and Ji (2015) ${ }^{22}$ concluíram que indivíduos com deficiência de vitamina D apresentaram maior risco para o desenvolvimento de DA e outros tipos de demência. 
Quadro 2. Estudos originais analisados.

\section{Estudos originais:}

\begin{tabular}{|c|c|c|c|c|c|}
\hline $\begin{array}{l}\text { Tipo de } \\
\text { estudo }\end{array}$ & Referências & Objetivo do estudo & População/Intervenção & Conclusões & NE \\
\hline 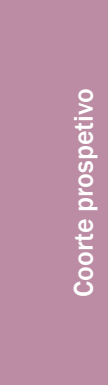 & $\begin{array}{l}\text { Karakis et al } \\
\text { (2016) }\end{array}$ & $\begin{array}{l}\text { Estudar a } \\
\text { associação entre } \\
\text { os níveis séricos } \\
\text { de vit. D e os tipos } \\
\text { de demência, } \\
\text { os marcadores } \\
\text { imagiológicos de } \\
\text { envelhecimento } \\
\text { cerebral e a função } \\
\text { neuropsicológica }\end{array}$ & $\begin{array}{l}\text { - } \mathrm{n}=1663 \\
\text { - Idade média } 72.4 \text { anos } \\
\text { - } \text { Follow-up } 9 \text { anos } \\
\text { - Medição dos níveis séricos basais de } \\
\text { vit. } \mathrm{D} \text { (deficiência < } 10 \mathrm{ng} / \mathrm{ml} \text { ) }\end{array}$ & $\begin{array}{l}\text { Não se observou } \\
\text { associação entre os } \\
\text { níveis séricos de vit. D e a } \\
\text { demência, em nenhum dos } \\
\text { modelos ajustados }\end{array}$ & 1 \\
\hline 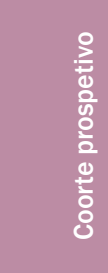 & $\begin{array}{l}\text { Moon et al } \\
2015\end{array}$ & $\begin{array}{l}\text { Estudar a } \\
\text { associação entre } \\
\text { os níveis basais de } \\
\text { vit. D e o risco de } \\
\text { desenvolver DCL } \\
\text { e demência em } \\
\text { idosos }\end{array}$ & $\begin{array}{l}\text { - } \mathrm{n}=412 \\
\text { - Idade média } 72,5 \text { anos } \\
\text { - } \text { Follow-up } 5 \text { anos } \\
\text { - Medição dos níveis basais de vit. D }\end{array}$ & $\begin{array}{l}\text { Níveis baixos de vit. D } \\
\text { estão associados com a } \\
\text { progressão de declínio } \\
\text { cognitivo e com o } \\
\text { desenvolvimento de DCL e } \\
\text { demência }\end{array}$ & 2 \\
\hline 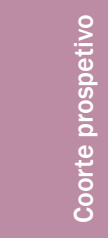 & $\begin{array}{l}\text { Knekt et al } \\
2014\end{array}$ & $\begin{array}{l}\text { Investigar se os } \\
\text { níveis séricos de } \\
\text { vit. D se relacionam } \\
\text { com a incidência de } \\
\text { demência }\end{array}$ & $\begin{array}{l}-\mathrm{n}=5010 \\
\text { - } 40-79 \text { anos } \\
\text { - Follow-up: } 17 \text { anos }\end{array}$ & $\begin{array}{l}\text { Verificada uma associação } \\
\text { inversa entre a } \\
\text { concentração sérica de vit. } \\
\text { D e a incidência de casos } \\
\text { de demência em mulheres } \\
\text { mas não nos homens }\end{array}$ & 1 \\
\hline 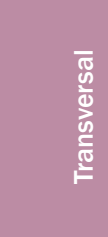 & $\begin{array}{l}\text { Vedak et al } \\
2015\end{array}$ & $\begin{array}{l}\text { Avaliar o papel da } \\
\text { vit. D no declínio } \\
\text { cognitivo }\end{array}$ & $\begin{array}{l}\text { - } \mathrm{n}=86 \\
\text { - }>50 \text { anos } \\
\text { - Follow-up: } 17 \text { anos }\end{array}$ & $\begin{array}{l}\text { Foram encontrados níveis } \\
\text { mais baixos de vit. D nos } \\
\text { pacientes com demência } \\
\text { quando comparados com } \\
\text { o grupo controlo e com o } \\
\text { grupo do DCL }\end{array}$ & 3 \\
\hline 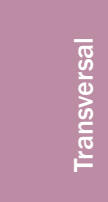 & $\begin{array}{l}\text { Prabhakar } \\
\text { et al } 2015\end{array}$ & $\begin{array}{l}\text { Avaliar a associação } \\
\text { entre os níveis } \\
\text { séricos de vit. D e a } \\
\text { demência vascular }\end{array}$ & $\begin{array}{l}\text { - } n=140 \text { (casos) } \\
\text { - } n=132 \text { (controlos) } \\
\text { - } \geq 65 \text { anos }\end{array}$ & $\begin{array}{l}\text { O défice de vit. D foi } \\
\text { associado a um risco } 2.2 \\
\text { vezes maior de demência } \\
\text { vascular, após ajustamento } \\
\text { de variáveis }\end{array}$ & 2 \\
\hline 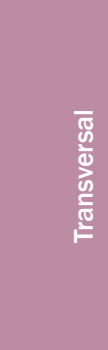 & $\begin{array}{l}\text { Yesil et al } \\
2015\end{array}$ & $\begin{array}{l}\text { Comparar os níveis } \\
\text { séricos de vit. D } \\
\text { entre indivíduos } \\
\text { com função } \\
\text { cognitiva normal, } \\
\text { DCL e DA }\end{array}$ & $\begin{array}{l}\text { - } \mathrm{n}=989 \text { (158 DA; } 228 \text { DCL; } 603 \\
\text { controlos) } \\
\text { - } \geq 65 \text { anos }\end{array}$ & $\begin{array}{l}\text { Os níveis de vit. D foram } \\
\text { estatisticamente diferentes } \\
\text { entre os } 3 \text { grupos. Houve } \\
\text { diferença estatisticamente } \\
\text { significativa entre o grupo } \\
\text { controlo e o DCL e entre } \\
\text { o grupo controlo e a DA, } \\
\text { com níveis mais baixos nos } \\
\text { segundos }\end{array}$ & 2 \\
\hline
\end{tabular}

Em dois estudos transversais ${ }^{23-24}$ foram encontrados níveis séricos mais baixos de vitamina $D$ nos pacientes com DA e demência, quando comparados com o grupo controlo. Também o estudo transversal de Prabhakar et al (2015) ${ }^{25}$ concluiu, após ajustamento de variáveis, que o défice de vitamina $D$ estava associado a um risco 2.2 vezes maior de demência vascular.
Relativamente aos estudos prospectivos, o estudo de Moon et al (2015) ${ }^{26}$, com follow-up de 5 anos de 412 indivíduos, concluiu que níveis baixos de vitamina $D$ estão associados com o desenvolvimento de demência. 0 estudo Knekt et al (2014) ${ }^{27}$, com seguimento de 5010 indivíduos ao longo de 17 anos, com o objetivo de investigar se níveis séricos de vitamina $D$ se relacionavam com a 
incidência de demência, concluíram existir uma associação inversa entre a concentração sérica de vitamina $\mathrm{D}$ e a incidência de casos de demência em mulheres mas não nos homens. Por último, na coorte de Karakis et al (2016) ${ }^{28}$, com um período de follow-up de 9 anos e 1663 participantes, não foi observada associação entre os níveis basais de vitamina D e o desenvolvimento de demência em nenhum dos modelos ajustados.

\section{Conclusão}

A evidência disponível mostra uma associação direta entre o défice de vitamina D e a demência (SOR B). Para além disto, parece haver associação positiva entre o défice de vitamina $\mathrm{D}$ e o risco de desenvolvimento de demência (SOR B). No entanto e, apesar desta evidência, é de ressalvar que apenas um dos dois estudos prospetivos de maior qualidade 27,28 incluídos nesta revisão mostrou uma associação entre a concentração sérica de vitamina $\mathrm{D}$ e a incidência de demência no sexo feminino.

Ainda não está completamente esclarecido o modo como a vitamina $\mathrm{D}$ e a demência estão associadas e se esta associação é causal. Parece haver duas principais interpretações. Por um lado, a demência poderá afectar a concentração de vitamina D. O declínio cognitivo e funcional associado a esta patologia, com subsequente perda de autonomia, pode dificultar a adequada exposição solar bem como uma alimentação com consumo adequado de alimentos ricos em vitamina D. Por outro lado, o défice de vitamina D poderá preceder o estabelecimento de demência. Esta hipótese é suportada pelo facto desta associação já se encontrar presente nas fases iniciais da demência e mesmo em casos de DCL, que não cursam com incapacidade funcional significativa.
De realçar que, apesar de a maioria dos idosos apresentar concentrações baixas de vitamina D, nem todos irão desenvolver demência. É portanto pouco provável que o seu défice explique o desenvolvimento de demência por si só, ainda que possa constituir um factor predisponente, com aumento da susceptibilidade à doença.

Salientam-se, contudo, as principais limitações metodológicas dos estudos incluídos: a grande variedade na idade dos participantes (sendo que participantes jovens poderão ter subestimado o efeito da hipovitaminose D na demência); a inclusão de participantes de ambos os sexos no mesmo estudo (sendo que parece haver respostas diferentes à vitamina $D$ em homens e em mulheres); o baixo tamanho amostral de alguns estudos; o uso de diferentes valores de referência na categorização dos níveis de vitamina D (com diferentes definições de hipovitaminose); os diferentes métodos de determinação das concentrações séricas de vitamina D; uma única medição das concentrações séricas de vitamina $D$ (que não reflete a variabilidade sazonal nem ao longo do tempo); o não ajustamento para variáveis confundidoras em alguns dos estudos; a suplementação com vitamina D em alguns dos controlos dos estudos; a existência de estudos com duração de follow-up potencialmente insuficiente.

A prevenção da demência é um assunto de grande importância em termos de saúde pública numa população em envelhecimento e, de acordo com os estudos realizados nos últimos anos, a hipovitaminose $D$ poderá ser um factor de risco modificável de demência. Desta forma, será importante a realização de ensaios clínicos prospetivos, bem desenhados, randomizados e controlados que, por um lado, permitam avaliar a existência de causalidade e, por outro, testem a eficácia e segurança da suplementação de vitamina $D$ na prevenção ou atraso no desenvolvimento de demência em populações idosas com hipovitaminose D. 


\section{Referências Bibliográficas}

1. Sampson EL. Palliative care for people with dementia. Br Med Bull 2010; 96:159-174.

2. Cherniack EP, Florez H, Roos BA, Troen BR, Levis S. Hypovitaminosis $D$ in the elderly: From bone to brain. J Nutr Health Aging 2008; 12:366-373.

3. Grant WB. Does vitamin D reduce the risk of dementia? J Alzheimers Dis 2009; 17:151-9.

4. Garcion E, Wion-Barbot N, Montero-Menei CN, Berger F, WionD. New clues about vitamin D functions in the nervous system. Trends Endocrinol Metab 2002; 13:100-105.

5. Neveu I, Naveilhan P, Baudet C, Brachet P, Metsis M. 1,25dihydroxyvitamin D3 regulates NT-3, NT-4 but not BDNF mRNA in astrocytes. Neuroreport 1994; 6:124-126.

6. Neveu I,Naveilhan P, Jehan F, Baudet C,Wion D, De Luca $\mathrm{HF}$, Brachet P. 1,25-dihydroxyvitamin D3 regulates the synthesis of nerve growth factor in primary cultures of glial cells. Brain Res Mol Brain Res 1994; 24:70-76.

7. Bouillon R, Carmeliet G, Daci E, Segaert S, Verstuyf A. Vitamin D metabolism and action. Osteoporos Int 1998; 8(2):S13-S19.

8. Cornet A, Baudet C, Neveu I, Baron-Van Evercooren A, Brachet P, Naveilhan P. 1,25-Dihydroxyvitamin D3 regulates the expression of VDR and NGF gene in Schwann cells in vitro. J Neurosci Res 1998; 53:742-746.

9. Naveilhan P, Neveu I, Baudet C, Funakoshi H, Wion D, Brachet $\mathrm{P}$, Metsis M. 1,25-Dihydroxyvitamin D3 regulates the expression of the low-affinity neurotrophin receptor. Brain Res Mol Brain Res 1996; 41:259-268.

10. Gezen-Ak D, Yilmazer S, Dursun E. Why vitamin D in Alzheimer's disease? The hypothesis. J Alzheimers Dis 2014; 40:257-269.

11. Holick MF et al. Evaluation, treatment, and prevention of vitamin D deficiency: an Endocrine Society clinical practice guideline. J Clin Endocrinol Metab 2011 Jul; 96(7):1911-30.

12. Palacios C, Gonzalez L. Is Vitamin D a major global public health problem? J Steroid Biochem Mol Biol 2014 October; 144PA:138-145.

13. Santos MJ, Fernandes V, Garcia FM. Carência de vitamina D numa população hospitalar: uma Fotografia pela Perspetiva Laboratorial. Acta Med Port 2015 Nov-Dec; 28(6):726-734.

14. Evatt ML, DeLong MR, Khazai N, Rosen A, Triche S, Tangpricha V. Prevalence of vitamin D insufficiency in patients with Parkinson disease and Alzheimer disease. Arch Neurol 2008;65:1348-52.

15. Sato Y, Asoh T, Oizumi K. High prevalence of vitamin D deficiency and reduced bone mass in elderly women with Alzheimer's disease. Bone. 1998;23:555-7.

16. Gu Y, Nieves JW, Stern Y, Luchsinger JA, Scarmeas N. Food combination and Alzheimer disease risk: a protective diet. Arch Neurol 2010; 67(6):699-706.

\section{Conflito de Interesses}

Os autores declaram não ter conflitos de interesses.
17. Etgen T, Sander D, Bickel H, Sander K, Forstl H. Vitamin $D$ deficiency, cognitive impairment and dementia: a systematic review and meta-analysis. Dement Geriatr Cogn Disord 2012; 33(5):297-305.

18. Annweiler C, Llewellyn DJ and Beauchet O. Low Serum Vitamin D Concentrations in Alzheimer's Disease: A Systematic Review and Meta-Analysis. Journal of Alzheimer's Disease 2013; 33:659-674.

19. Cao L, Tan L, Wang H, et al. Dietary Patterns and Risk of Dementia: a Systematic Review and Meta-Analysis of Cohort Studies. Mol Neurobiol 2015.

20. Schaft JVD, Koeka HL, Dijkstraa E, Verhaar HJJ, Scouwb YT, Emmelot-Vonka MH. The association between vitamin $\mathrm{D}$ and cognition: A systematic review. Ageing Research Reviews 2013; 12:1013-1023.

21. Silva SL, Vellas B, Elemans S, et al. Plasma nutrient status of patients with Alzheimer's disease: Systematic review and meta-analysis. Alzheimer's \& Dementia 2014; 10:485-502.

22. Shen L, Ji HF. Vitamin D deficiency is associated with increased risk of Alzheimer's disease and dementia: evidence from meta-analysis. Nutr J. 2015; 1:14:76.

23. Vedak TK, Ganwir V, Shah AB, et al. Vitamin D as a marker of cognitive decline in elderly Indian population. Ann Indian Acad Neurol. 2015; 18(3):314-319.

24. Yesil Y, kuyumcu ME, Kara O, et al. Vitamin D status and its association with gradual decline in cognitive function. Turk J Med Sci 2015; 45:1051-1057.

25. Prabhakar P, Chandra SR, Supriya M, Issac TG, Prasad C, Christopher R. Vitamin D status and vascular dementia due to cerebral small vessel disease in the elderly Asian Indian population. Journal of the Neurological Sciences 2015; 359:108-111.

26. Moon JH, Lim S, Hant JW, et al. Serum 25-hydroxyvitamin $\mathrm{D}$ level and the risk of mild cognitive impairment and dementia: the Korean Longitudinal Study on Health and Aging (KLoSHA). Clinical Endocrinology 2015; 83:36-42.

27. Knekt P, Saaksjarvi K, Jarvinen R, et al. Serum 25-hydroxyvitamin d concentration and risk of dementia. Epidemiology 2014; 25:799-804.

28. Karakis I, Pase MP, Beiser A, et al. Association of Serum Vitamin D with the Risk of Incident Dementia and Subclinical Indices of Brain Aging: The Framingham Heart Study. J Alzheimers Dis 2016; 51(2):451-461.

29. Muscogiuru G, Altieri B, Annweiler C, et al. Vitamin D and chronic diseases: the current state of the art. Arch Toxicol 2016.

30. Annweiler C, Dursun E, Féron F, et al. 'Vitamin D and cognition in older adults': updated international recommendations. J Intern Med 2015; 277:45-57.

\section{Endereço para Correspondência:}

Maria Margarida Baldaque de Sousa Soares da Silva Oliveira E-mail: 